\title{
Designing an Optimal Model of Blood Logistics Management with the Possibility of Return in the Three-Level Blood Transfusion Network
}

monireh Ahmadimanesh ( $\nabla$ monireh.ahmadimanesh@yahoo.com )

Sadjad University of Technology https://orcid.org/0000-0003-0836-1620

\section{Alireza Pooya}

Ferdowsi University of Mashhad Faculty of Economics and Business Administration

Hamidreza Safabakhsh

Iranian Blood Transfusion Organization

Sedigheh Sadeghi

Ferdowsi University of Mashhad School of Mathematical Science

\section{Research Article}

Keywords: Blood logistics management, three-level blood transfusion network, simulation, neural network

Posted Date: January 4th, 2022

DOI: https://doi.org/10.21203/rs.3.rs-1187827/v1

License: (c) This work is licensed under a Creative Commons Attribution 4.0 International License.

Read Full License 


\title{
Designing an Optimal Model of Blood Logistics Management with the Possibility of Return in the Three-Level Blood Transfusion Network
}

\begin{abstract}
:
Inventory managers in the blood supply chain always seek timely and proper response to their customers, which is essential because of the perishability and uncertainty of blood demand and the direct relationship of its presence or non-presence with human life. On the other hand, timely and regular delivery of blood to consumers is vital, as the weakness in delivery and transportation policies results in increased shortages, returns, blood loss and significant decrease in the quality of blood required by patients. Given the significance of this for the blood transfusion network, the paper tried to design a comprehensive and integrated optimal model of blood transfusion network logistics management by blood group to reduce the cost of losses, returns and blood shortages. This model is divided into two parts: Inventory management and routing. A combination of simulation techniques and neural network with several recurrent layers was used to evaluate the optimal inventory management and a multi-objective planning model was designed to determine the delivery and distribution of blood to consumers. The model designed was implemented in Khorasan Razavi Blood Transfusion Network with a main base, six central bases and 54 hospitals. Solving the model led to estimating the $\mathrm{f}$ consumer demand, the optimal value of target inventory and reordering point of central bases and hospitals, and blood distribution from the supplier to its consumers that decreased the units of blood returned to bases, increased inventory availability, and reduced costs significantly.
\end{abstract}

Keywords: Blood logistics management, three-level blood transfusion network, simulation, neural network

\section{Introduction}

Blood transfusion is one of the branches of medical sciences with a significant and undeniable role in providing medical services to the patients and its position in the country has to be seriously taken into account by health policy makers and planners. Regarding this, blood supply managers must always try to create enough reserves to increase access to different blood products and reduce waste because of the expiration of blood expiration time to meet the challenge of waste and lack of blood supply chain. The blood products consumed in hospitals is a function of the number of daily incidents with an uncertain nature, and this makes it possible to match, in most cases, the exact predicted value of daily demand in hospitals with the value of practical demand. Under such conditions, hospitals prefer to order more blood products to have a safe stock to deal with possible deficiencies, yet over-ordering blood products by hospitals is a significant issue due to the limited amount of blood in the regional blood center or blood transfusion bases [1]

Blood bank inventory management can be discussed at three levels: the individual hospital level (Hospital Blood Bank)-HBB, The regional level (Regional Blood Bank)- 
RBB and general blood transfusion organization. Among these levels, RBB inventory management will be more critical, as RBB management is naturally more complex than HBB management, because of the large number of bases and hospitals in regional systems, the high frequency of ongoing activities and complexity of organizational structure [2,3]. This is because the decision-maker is the director of the regional blood center and formulates distribution policies between hospitals and manages matters such as planning the collection, distribution and transmission of blood in the region. Thus, the success of RBB management, depending on the use of optimal and efficient methods in planning the level of blood inventory threshold, forecasting consumer demand, formulating policies for the collection, storage and ordering of blood Consumers seek to respond in a timely manner to patient demand [4]. On the other hand, a RBB is faced with the situation that a set of customers (hospitals, clinics, and medical institutions) need regular deliveries of certain products that make them consume different substances fast. Any delivery policy has to be such that there is no shortage of products for the consumer and at the same time product corruption occurs to a minimum [5]. Because of the existence of such conditions, proper transportation of blood and its products is one of the important steps to maintain the quality of blood needed by patients. Vehicle Routing Problem (VRP) can be used to solve this problem. The purpose of the routing problem in the simplest and most common case is to minimize the overall cost of the route based on the maximum working time and maximum capacity of vehicles while maintaining the quality of products [6]. Overall, one can state that one of the most important challenges of the blood transfusion organization is the preparation, distribution and monitoring of proper consumption of blood and blood products. Regarding this, the Blood Transfusion Organization has to try to bring about the possibility of blood supply and its products for all patients in nationwide while monitoring the blood reserves in the region on a daily, monthly and annual bases and the operation of the blood supply network.

Considering the sensitivities expressed, the need for studies according to enhancing inventory management and blood distribution in blood transfusion sites is felt. Thus, in the present paper, we tried to design and test a model that

- Firstly, it is comprehensive and integrated, applicable to all suppliers and consumers present in a regional blood bank (including several bases and hospitals).

- Secondly, it can optimize inventory management and blood distribution in the region simultaneously.

It has to be noted that as the highest demand and consumption for red blood cells is among the components of blood, the study provided appropriate and scientific tools and techniques for designing an inventory management model and red cell distribution by four principal blood groups $(\mathrm{A}, \mathrm{B}, \mathrm{O}, \mathrm{AB})$ in a $\mathrm{RBB}$ and attempts have been made to formulate optimal inventory management and distribution policies.

\section{Literature review}


The scholars in the field of supply chain have had a main role in developing effective models and methods for managing the stock of perishable goods [7]. A significant part of the relevant literature was developed from 1970 to 1980. The first framework for classifying the blood supply problem was proposed by [8]. This initial work presents the problem through a hierarchical level (strategic, technical and operational) and indicates the effect of the application of various blood supply policies.

[9] have analyzed the inventory management policies of a UK-specific blood supply system through a regional blood center. [10] developed integer programming models to decide which hospitals a vendor should visit each day (through blood center vehicles). In a blood study, [11] searched to solve the problem of allocating and routing blood to the public and private health systems. They modeled the problem as multi-criteria allocation routing problems. The models were solved by the method of hierarchical analysis and correct linear programming. The quality of both models was evaluated with real data. [12] combined the dynamic random scheduling and simulation to present the inventory management model of the Dutch Regional Blood Bank. [13] has presented a simulation model that fits the operational characteristics of the blood center inventory system. By implementing this model based on the historical data of the Regional Blood Bank of Jiangsu Province from 2005 to 2008, he has made recommendations for inventory management. [14] have developed a public network optimization model for the complex human blood supply chain using the multivariate optimization method. For this purpose, it has considered a regional blood bank system that includes collection, testing, processing and storage facilities, and distribution centers, and has tried to reduce scarcity and waste implement cost by implementing the model. This model has been solved with numerical examples and the optimal values of each cost were determined. In their paper, [15] showed the modeling and simulation of blood collection for collection bases in urban and rural settings in France. [16] used a discrete event model to analyze and propose inventory policies at RBB Center. The model shows the activities performed in the supply chain such as blood collection, testing, production, and inventory management. Using a reusable simulation model, [4] developed and evaluated regional blood network inventory policies in Canada using the level of response method and nonlinear planning to formulate optimal available inventory policies / supplier. [17] presented a vehicle routing model for blood bank logistics. In their paper, they addressed the issue of distributing and collecting blood for a general medical system as the problem of vehicle routing with delivery and collection. To this end, they used a heuristic model, a combination of genetic algorithm and simulation. [18] carried out a study at the Icelandic Blood Bank, which has a distribution center and a processing center. The study used discrete simulation to determine the optimal ordering policy to enhance the blood supply chain and reduce the level of waste. Moreover, to better understand the blood supply chain, linear regression analysis was used to explain how the chain can be affected. The results of 
the optimal reserve level model showed the shortage in a given period and the fluctuations in the inventory. [19] suggested a solution method for the management of red blood cells in the treatment center in the regional network that can be used in practice. The purpose of designing this model and combining it with dynamic planning was to reduce costs in the inventory management system of perishable goods and reduce the losses of blood centers. The results of solving the designed model with numerical examples determine the best storage and ordering policy. [20] used artificial neural network (ANN) technique to predict blood demand in blood transfusion centers. They predicted the monthly demand for three components of blood (red blood cells, plasma, and platelets) in an RBB. [21] et al. (2020) used neural network and reusable simulation to design an optimal inventory management model in an area without separation of blood units. The results showed a significant reduction in loses and deficiency of blood units. [22], studied the problem of blood routing in uncertainty conditions and discussed its unique features that distinguish a problem from traditional routing. For modeling the programming, they proposed a hybrid linear integer to solve the blood routing problem. [23] examined the problem of blood routing from blood centers to some hospitals under uncertainty conditions using two models of combined integer programming and probabilistic robust planning. Data from a real supply chain was used to solve these models. [24] suggested a routing inventory model for perishable goods with high consumption rates. In this model, they looked for determining the production per day, the sales centers that should be visited every day, the route of visits to the sales centers and the goods delivered to the sales centers per visit. The proposed model was solved with a numerical example that included a manufacturer node and three sales centers. According to the solution, the value of the objective function was 15729.88. Table 1 is the summary of the past studies in the two areas of blood supply chain and logistics, inventory management, and RBB transportation.

A review of previous studies shows several research gaps. There is considerable literature on blood inventory management and distribution separately, but few papers have examined large-scale regional blood transfusion networks. Moreover, most of the studies on RBB have proposed a model solved without implementation and only with numerical examples or implemented in a limited blood bank. Ultimately, in the studies examined, an integrated and comprehensive model of inventory management and distribution in the area applicable to all suppliers has not been designed. What distinguishes the present study from others in blood supply chain and logistics is that it designs an optimal model of inventory management and blood distribution in the regional network. Among the partial features of the model, one can say that firstly, the optimal model of inventory management designed with the help of two techniques of simulation and neural network is comprehensive and general and used for all available bases (suppliers) on the network and there is no need for any supplier in the region to 
implement an optimal model of inventory management to re-modeling and spending cost and additional time, and only with the update of events can be developed by current and desirable behaviors. Moreover, in this part of the model, the correct customer demand is estimated and the optimal target inventory and re-order point is determined for all suppliers and consumers in the region. In other words, besides significantly assisting suppliers in estimating demand and deliveries, this technique will bring about the optimization of consumer ordering policies. Secondly, the multiobjective programming routing model is associated with blood inventory management variables and the implementation of this model leads to the optimization of the distribution of blood units to the desired hospitals with the least losses, reducing the time of blood transfer to consumption, increase delivery speeds and reduce the accumulation of distribution machines at supplier locations.

\section{Description of the model}

The study tries to optimize the inventory and distribution in the blood logistics chain in a region. The region has three levels of regional blood transfusion organization, central bases and hospitals as shown in Figure 1. In this regional model, which has a more flexible organizational structure, the functional parts and part of the coordination / cooperation between the $\mathrm{CBCs}$ are performed by the regional blood center or the main base (RBC) and the activities Coordinates and controls the collection of blood centers in a region. $\mathrm{RBC}$ provides services like information processing and training programs, donor recruitment, etc., and is considered as the decision maker and authority for the operation of the entire system.

The first part of the model, implemented at the level of regional blood transfusion organizations in the region, uses a combination of simulation algorithm and neural network. The output of this algorithm is the inventory control policy $\mathrm{S}$ (target inventory value) and $\mathrm{R}$ (order point) that determines and obliges the whole region to implement it. After communicating the type of inventory management system to the lower levels, they need to implement this policy (Figure 2, RBC level). At the second level, the director of the blood bank of each central base is required to decide on sending the best amount of blood they need and to use the specified amounts, according to the inventory of his central base and the total demand of the hospitals under their coverage and send their subscribers according to the routing plan and using the multi-objective planning technique (Figure 2, CBC level). As consumers, the hospitals add blood to their inventory after receiving it from the truck, and after transferring it to patients, compare the end of the remaining inventory with the reordering point and decide to order the next day (Figure 2, hospital level). Figure 2 is the research algorithm.

Reaching each of the steps of the optimal model of inventory management and distribution as three levels $\mathrm{RBC}, \mathrm{CBC}$ and hospital is described below. 


\subsection{Designing inventory management model by RBC Organization}

The purpose of designing the inventory management model by $\mathrm{RBC}$ is to determine the target inventory values and the ordering point of each supplier and consumer in the network to minimize the amounts of deficiencies and wastes in the system under study. Thus, a simulation technique has been implemented at the $\mathrm{RBC}$ level to design this model, the output of which will result in instructions for the CBC that specify the target inventory values and the ordering point in each supplier. Using MATLAB software, a database was used to store the parameters related to the implementation of the simulation model and to record the output results. As the input of the simulation model needs demand information, it is essential to properly estimate consumer demand, which is done with a neural network. Hence, first, forecasting consumer demand is explained, and then the simulation flowchart is explained.

\subsubsection{Estimating consumer demand using neural network}

Implementing the inventory model with the simulation technique really needs the daily demand of each consumer. Thus, it was necessary to first calculate the value of demand of each consumer to provide other steps of the inventory management model of the desired inventory values. In other words, the technique compares what is recorded as consumer demand in the current inventory management system with what should actually be sent. The technique used in this section is a neural network with several return layers and fully connected. At this step, network parameters, net input, transfer function, learning function, learning rate and the number of neurons in each layer were determined. Then the data was normalized and standardized for preprocessing. In the next step, the data was divided into three parts: training, test and validation data. Ultimately, the neural network was implemented and the necessary corrections were made to reach the best outcomes. The demand of each consumer was obtained from Equation 1 and entered the neural network as the input layer variable. This equation causes the value of returns to be included in the calculations as the value of fines and prevents the expiration of blood units due to non-use. The output layer predicts the daily demand for red blood cells. The hidden layer has the proper number of neurons, selected according to mean squared error (MSE). The input layer, the hidden layer and the output layer are completely connected. Connection weights are randomly selected by the system and adjusted through the learning process. The sigmoid function in the hidden layer is considered according to Equation 2 to calculate the sum of the weights in the range $[-1,1]$. Thus, the transfer function in the latent layer and the output layer for each type of red blood cell are sigmoid function and linear transfer function, respectively.

$$
A_{t i r}=D_{t i r}-C_{t i r}-O_{t i r}
$$


$D_{\text {tir: }}$ Hospital $\mathrm{i}$ blood request for blood group $\mathrm{r}$ at time $\mathrm{t}$

$\mathrm{C}_{\text {tir: }}$ : The returns due to non-consumption of hospital i for blood group $\mathrm{r}$ day $\mathrm{t}$

Otir: The rate of return due to hospital expiration i for blood group $\mathrm{r}$ day $\mathrm{t}$

Atri: Consumer i demand for blood group $r$ at time $t$

$f(t)=\frac{2}{1-e^{-2 t}}-1$

In the next step, the function of distributing the estimated demands of hospitals and the value of consumption (demand) of each central base, the range of target inventory values and the point of re-ordering of each central base and hospital and the useful life of products are entered into the simulation algorithm. The average weekly (daily) demand of each central database and hospital has been calculated separately by blood group and equal to $\pm 20 \%$ of this value is considered as the maximum and minimum range to determine the range of target inventory values of each database. Moreover, to determine the range of re-ordering points of each database, their inventory information was attained on a daily basis when ordering in one year, and the average daily reordering point of each central and hospital base by blood group was calculated \pm 5 of this value was considered as the maximum and minimum interval value. It has to be noted that the desired intervals have been entered into the simulation algorithm, and the interval is reduced if during the execution the optimal value of the proposed algorithm is on the minimum interval value, and the interval is considered larger if it is on the maximum interval value.

\subsubsection{Determining the optimal ordering policy of $\mathrm{CBC}$ level and hospitals using simulation}

It is necessary to determine the number of iterations and the length of repetitions of each performance in each iteration to perform the simulation technique, according to the distribution of demand or consumption of hospitals and central bases, various demands are produced and measured from Equation 3 (formula t-test) was used to calculate the number of repetitions of each simulation run. Based on this equation, the error percentage numbers (B) and standard deviation (s) are determined and based on the $\mathrm{t}$-test formula, the value of the variable $\mathrm{n}$ (number of repetitions of each execution)

is determined. $\overline{T C}$ is the average value of the objective function (minimizing the number of wastes and deficiencies).

$$
\left[\overline{T C}-t_{n-1,1-\frac{B}{2}} \frac{s}{\sqrt{n}}, \overline{T C}+t_{n-1,1-\frac{B}{2}} \frac{s}{\sqrt{n}}\right]
$$

Based on the error rate of $5 \%$ and the standard deviation considered, the number of iterations of each execution is 20 iterations. Thus, each simulation run was carried out with 20 iterations in one year (iterations length). The implementation algorithm of each simulation run is carried out separately for each central base and hospital and based on each blood group according to Figure 2. The upper part of this figure is responsible for inventory control and responding to demand. According to the algorithm shown in 
Figure 2, the customer enters the system with a specific distribution function. When the customer enters, one has to examine whether there is an inventory or not? If ILri> is not 0 , one unit is added to the number of missing values of one unit. However, if ILri> 0 , units of blood are assigned to the customer (deplet). Deplet ++ means that at this stage, the number of customers whose demand has been met is calculated. When blood units are removed from the system, one has to check whether the instantaneous inventory level ( $\mathrm{IL}_{\mathrm{ri}}$ ) has reached $\mathrm{R}_{\mathrm{ri}}$ level or not. If it does not reach $\mathrm{R}_{\mathrm{ri}}$ level, no action is taken (dispose). Nonetheless, if the current inventory level has reached $R_{r i}$ level, an order must be placed for the inventory level to reach Sri. In this case, it is added to the number of blood orders. The lower part of the algorithm is responsible for removing perished blood from the system whenever the product life is over. In this section, inventory managers enter the system at least once a day and remove corrupt materials from the system and check how much blood has been consumed by customers and how much has expired from the target inventory (The algorithm determines that once the inventory level reaches $S_{r i}$ value. It is determined by giving the product longevity time, every few days the inventory manager enters the system and removes expired products).

Thus, according to the algorithm in Figure 3, a continuous inventory policy is defined for the hospitals and central bases in the system. After determining the target inventory values and the reordering point in each of the central bases and hospitals, the system is simulated for one year so that whenever the inventory level of each role reaches the reordering point $\mathrm{r}_{\mathrm{i}}$, the inventory system of that role (Supplier or Consumer) raises its inventory level to the target inventory level $\mathrm{S}_{\mathrm{i}}$ - orders the value of $\mathrm{S}_{\mathrm{i}} \mathrm{r}_{\mathrm{i}}$ (such as $\mathrm{r}_{\mathrm{i}} \leq \mathrm{S}_{\mathrm{i}}$ ). The simulation algorithm examines all given intervals and provides the best target inventory and reorder point for each consumer and supplier role so that the value of waste and shortage in the whole system is minimized. Ultimately, the last element of the algorithm stores the data to control the model and acts as a repository for information output from the simulation. Table 2 is the symbols used in the simulation technique.

\subsection{Implementation of inventory management model on a daily basis in central bases and hospitals}

After notifying the target inventory values and the reorder point to the central databases, they are obliged to implement the inventory management model according to the notification instructions. Consecutive steps are taken from supplier to consumer every day to implement the inventory management model by them. At the start of the day, the blood bank resource manager of the supplier calculates the value of blood bank reservoir balance. This value of inventory includes the total of healthy blood collected in the donation bases (mobile and fixed) sent to the central base for 
performing the desired tests, the remaining blood from the previous days. Residual blood from previous days that have a shelf life, surplus blood received from other central bases received at the start of the day, blood received from the consumers with extra inventory and sent to the returned supplier tank is deducted from the value of blood in the tank with their expiration date has expired. In the next step, the value of demand of each consumer is received separately (the demand is received from $\mathrm{RBC}$ for the first time, but for the next time, the order is directly taken from the consumer). After calculating of the total demand received, the $\mathrm{CBC}$ Inventory Manager examines whether the total demand is less than the target inventory announced by the RBC. If all requests have been answered and have not yet reached the target balance, all requests will be answered. Nonetheless, if the value of the requests exceeds the value of the target inventory, the inventory manager must calculate the value sent to each consumer. It will distribute the blood among the consumers according to the routing model to send consumers' blood to increase the delivery speed and reduce the shipping cost. This is done according to multi-objective planning as described in Section 3-2-1. After sending the requests to the consumers based on the routing model, if there are emergency orders by the end of the day, it will respond to them and at the end of the day, it will calculate the blood bank balance again. If the remaining balance at the end of the day is higher than the target inventory, it sends it as a surplus to other central inventories in the network, and it requests blood from other central bases if the remaining inventory has reached the re-order point. This is where the supplier day (central base) ends. Simultaneously with the provider of consumer behavior (hospitals) in a day is planned as follows. After receiving the blood needed from the supplier, it adds it to its inventory and consumes it gradually during the day. At the end of the day, the hospital blood bank manager calculates his balance. If the blood bank has more than the target balance announced by the central bases, it is considered as a surplus and sends it to its supplier to prevent it from increasing and becoming a perished commodity (Commodity return). However, it is checked whether this inventory has reached the re-order point or not if the current inventory is less than the notified target inventory. If it reaches the re-order point, it will determine the desired order quantity for the next day; otherwise, no action is taken and the day ends.

\subsubsection{Designing distribution management model}

A routing model was developed to optimize the transport status of blood logistics chain. With the implementation of this model, each central base will have more control over the distribution of blood and will prevent possible waste in the truck and the sudden accumulation of applicant machines. Thus, the purpose of this step is to specify the optimal route model with the least cost and loss. The routing model used in the study was a multi-objective programming mathematical model whose symbols are given in Table 3. The assumptions and specifications of the model are as follows:

- There is blood exchange between central bases (CBCs) in the logistics network. 
In a logistics network, a supplier delivers blood to multiple consumers (hospitals under its cover and other central bases).

- It is impossible to exchange blood units between hospitals.

The supplier uses a heterogeneous transport network (fleet) with vehicles that differ in capacity and cost to distribute blood to consumers.

- Each blood unit has a specific expiration date and after that it will be unusable.

- It is possible for consumers to return expired or unused blood.

Consumer requests have to be submitted within a specified time frame.

- The inventory capacity of each consumer for each blood is recognized by the supplier.

- A cost of shortage penalty is considered for the supplier because of the high degree of uncertainty in the demand value.

- The level of initial blood supply in each consumer is known.

- Precautionary reserve is specified for each supplier and consumer.

In the model designed, the first objective function tries to minimize transportation costs. The mathematical form of this function is expressed in Equation 4. The second objective function, which is given in Equation 5, minimizes the total distance traveled. Constraint 6 ensures that the capacity of each vehicle should not be too specific. This constraint implies that the starting point of each vehicle has to be the supplier (central bases). Equation 7 is the turning of vehicles. Equation 8 is for removing the sub-tour in the route graph so that a vehicle does not return to a consumer. This constraint shows that if the vehicle is to go from consumer $i$ to consumer $j$ and serve them (there is a connection between consumers $i$ and $j$ in direction $i$ to $j$ ), $w_{j k t}$ has to be greater than $w_{i k t}$ so that the vehicle does not serve a single consumer more than once and does not create a subtour in the graph path. Equations 9 and 10 show the decision variables.

$$
\begin{gathered}
\operatorname{Min}_{1}=\sum_{(i, j) \in M^{\prime}} \sum_{k \in K} \sum_{t \in T} \tilde{v}_{i j k} y_{k t} x_{i j k t} \\
\operatorname{Min}_{2}=\sum_{(i, j) \in M} \sum_{k \in K} \sum_{t \in T} c_{i j} x_{i j k t} \\
\sum_{r \in R} \sum_{i \in M} \sum_{t \in T} q_{i r k t} \leq c a p_{k} y_{k t} z_{0 k t} \quad \forall k, t \\
\sum_{j \in M^{\prime}} x_{i j k t}=\sum_{j \in M^{\prime}} x_{j i k t}=z_{i k t} \quad \forall i \in M^{\prime}, k, t \\
w_{i k t}-w_{j k t}+(|M|+1) x_{i j k t} \leq M \quad \forall(i, j) \in M, k, t \\
x_{i j k t}, z_{i k t} \in\{0,1\} \quad \forall i, j \in M, k, t, i \neq j
\end{gathered}
$$


$q_{i k t}, I_{\text {irt }}, E X_{\text {irt }}, S H_{\text {irt }}, y_{k t} \geq 0, \quad$ Integer $\forall i \in M, r, k, t$

The routing model, as a multi-objective planning model for minımızing transportation costs and reducing the distance traveled, tries to determine the best route of blood transmission to consumers with the possibility of returning blood to suppliers. The model was solved in Cplex software and the Epsilon method was used to convert the multi-objective to single-objective function.

\section{Case study}

The proposed model of the study was implemented and run in the blood transfusion network of Khorasan Razavi with the structure according to Figure 4. Based on this figure, Khorasan Razavi province has a main base in the center of the province, six central bases in Mashhad, Sabzevar, Torbat Heydariyeh, Neishabour, Gonabad and Qouchan and 54 hospitals. Each central base receives the blood collected from the mobile and donated bases and after performing the necessary tests on them and separating the blood components, stores and packages it as the central base inventory to distribute to its consumers if needed. Coordinating and organizing the central bases is done by the main base (regional blood center) in Mashhad, which monitors their activities. Daily data available in blood transfusion databases of Khorasan Razavi from September 2015 to September 2017 was used to implement the developed model.

\section{Results}

The proposed study model was implemented and run in a comprehensive and integrated way in the blood transfusion network of Khorasan Razavi. As the model used in the study has two main parts of inventory management and routing, the results are obtained separately for each central base of the province and based on the two main parts of the model. However, this part only states the results of the central base. Mashhad (as the largest base). The base continuously serves 27 hospitals in Mashhad and six hospitals in the cities of the province that do not have a central base.

\subsection{The results of implementing optimal inventory management model for Mashhad central base}

\subsubsection{The results of estimating the demand of hospitals in Mashhad central base}

Then 500 days of data were used for training and validation of the neural network and 230 days for network testing from among the total data available in the period of September 2015 to September 2017 for each type of red blood cell. Various network architectures with the number of hidden layers (between 2 to 15 neurons), three input variables, and one output variable were examined and finally, the model with the lowest MSE value was selected (Table 4) to select the most appropriate neural network model to estimate the blood demand of each user by blood group. According to the neural network technique, first the real demand of all users of this database was estimated separately by blood group and then the demand of each consumer was calculated separately to receive more accurate information. Figure 5 is 
the total consumer demand by blood type and Figure 6 shows the demand of Aria Hospital as a sample consumer of this database.

The red lines in Figure 5 indicate the optimal and real demand of consumers from the central base of Mashhad. The results in this figure show that if the hospitals of Mashhad intended to order the optimal value to prevent shortages and spoilage, they must request the needed blood units according to the red lines. The blue lines show the value of blood requested by all hospitals in Mashhad from the central base of Mashhad in each blood group. The difference between the blue and red lines shows the gap between the current and the desired states. As various blood types show, what has actually happened is that all the hospitals in Mashhad have requested blood far beyond their needs, which has led to significant returning waste due to expiration in the system. The blue lines were much larger than the red lines on some days, showing a lot of blood loss throughout the logistics chain. This much error and the difference between what was supposed to be requested and what actually happened in the rare blood type $\mathrm{O}$ will result in irreparable damage. Because of the large number of consumers, the database is more sensitive than other databases in the region; thus, there is a strong need for optimal inventory planning and strict monitoring of its consumers.

What can be understood from the results of Figure 6 is that in fact, the requests of Aria Hospital in some days were much more than they needed and, in some days, much less, leading to waste and many shortcomings in the whole system. The results of the figure show that if the central base of Mashhad wanted to have the lowest rate of return and shortage from Aria Hospital, it should have sent blood units according to the red lines.

5.1.2. The results of the target inventory of Mashhad central base and the hospitals covered by it

According to the demand distribution function of Mashhad central base and its consumers, as well as the shelf life of the sent bloods, the simulation technique has determined their target inventory with the minimum waste and shortage with the results of Mashhad central base and several hospitals given as an example in Table 5. In other words, each central base and hospital has the maximum inventory it can hold that ends in the least waste and shortage.

\subsubsection{Reorder point}

One of the outputs of the simulation algorithm is to calculate the reorder point of each base and its consumers by considering the minimum waste and return. Table 6 is the values of the re-ordering point of Mashhad central base and some examples of its consumers.

\subsection{The results of implementing the routing model of Mashhad central base}

According to the inventory data at the start of the period, the actual demand estimated by the neural network, the value of target inventory and the re-ordering point of the output of the distribution and routing simulation output for seven days of Mashhad central base were compiled. In doing so, seven days were considered from October 
2015 to October 2017, and according to the Mashhad Central Base inventory at the start of the period, the estimated real demand of the consumers of this base in those seven days (neural network output), the value of target inventory and the point of reordering it and its consumers, the daily planning of the base was done for seven days and a routing plan was developed. Solving this model is done in Cplex software with specifications on a PC Pentium Corei5, 2.27 GHZ with 4.0 GB RAM. In doing so, the vehicle information obtained from the distribution base of the main base is presented as in Table 7.

The planning period in this model is seven days, where the distribution route of the consumers of this base (consumers of Mashhad and the cities) stated for the first day of the planned period in Table 8 .

\section{Validation}

As the model is divided into three sub-stages, the validation is carried out separately for each stage as described below.

Firstly, 500 days of data were allocated for training, and 230 days of data for testing in the demand estimation phase. The neural network structure was designed and coded so that it validated the data during the training to validate the model, so the neural network technique was validated during 500 days of training. Statistical analysis was used to evaluate the performance of the model. Statistical parameters including mean relative absolute error, mean absolute error, $\mathrm{R}^{2}$ constant and mean squared error were used for this analysis. Table 9 is the statistical error analysis of the neural network model. As is seen, the model has high accuracy in all three steps of training, validation and testing.

In the second step, to validate the simulation algorithm used, the re-ordering point and the inventory of consumers and suppliers were received within one year time, and the waste and shortages in this period were simulated and extracted by the simulation algorithm with these order values and compared with the actual waste and deficiencies in the period. It has to be noted that the data available in the central database of Mashhad has been examined as a sample to test the validity of the model as the proposed model has been implemented in six central databases and 54 hospitals. For this purpose, it was first found that the variances of the two communities (output of simulation results and data from real system history) are not the same using $\mathrm{F}$ test. The, at $95 \%$ confidence level, knowing the non-equality of the variances on the real sample data and the simulation model with the assumption of zero that the mean of both populations were equal, an independent t-test was performed and according to the results as stated in Tables 10 and 11, the value of $\mathrm{p}$ is considered an acceptable value and the null hypothesis was confirmed. It meant that the mean of the two populations was equal. The results show no significant differences between the simulation results and the actual data. Thus, the results of simulating the behavior of bases and hospitals can be trusted. 
In the third step, ten various numerical samples with similar conditions to the problem hypotheses are solved by the model to validate the multi-objective planning model, whose results show the validity of the model.

\section{Discussion}

The purpose of the study is to design an optimal model of inventory management and blood distribution in the regional network of blood transfusion in Khorasan Razavi. In doing so, the demand of hospitals as consumers is estimated using ANNs and the optimal behavior of all actors in the region (central bases as suppliers and hospitals were identified as consumers) with the help of the reusable simulation process, which will lead to a significant reduction in returned blood units by consumers, optimal management of suppliers and consumers to prevent waste and shortages. Then the designed model was examined for reducing costs, increasing the speed of transportation and the optimal distribution of blood requested by consumers using the routing method. The model suggested of real hospital demand is extracted and then the inventory of central bases is managed based on its information. What distinguishes this model from other models suggested in previous studies is its applicability in one region so that it is not necessary to write a separate model for each base in the region. Thus, with the existing algorithm, one can update events and develop current and desirable behaviors. The research team suggests that assumptions like potential demand, consideration of blood waste, and using a fuzzy approach to the routing model should be considered in this model for future studies to complete the strength of the model in the study. 


\section{References}

1. Yaghoubi, S., Kamur, (2017). Consumption management of products in the blood supply chain by considering the possibility of lateral transfer between hospitals (Case study: Tehran), Journal of Industrial Management Studies, Fifteenth Year, No. 47, pp. 93-119.

2. Lowalekar, H., Ravichandran, N(2013). Blood bank inventory management in India, Operational Research Society of India.

3. Prastacos ,Gregory P. (1984), Blood inventory management: An overview of theory and practice, management science, Vol. 30, No. 7, July 1984

4. Blake, J.T., Hardy, M. (2014). A generic modelling framework to evaluate network blood management policies: The Canadian Blood Services experience. Operations Research for Health Care 3,pp. 116-128

5. Hemmelmayr V, Doerner K F, Hartl R F (2009) A variable neighborhood search heuristic for periodic routing problems. European Journal of Operational Research, 195:791-802

6. Soysal, Mehmet. Ruwaard, Bloemhof. Jacqueline M (2016). Modeling a green inventory routing problem for perishable products with horizontal collaboration, Computers and Operation Research

7. Nahmias, Steven, and William P. Pierskalla. 1976. "A Two-product Perishable/Nonperishable Inventory Problem." SIAM Journal on Applied Mathematics 30 (3): 483-500.

8. Jennings, John B. 1973. "Blood Bank Inventory Control.” Management Science 19 (6): 637-645

9. Katsaliaki, K. Brailsford, S.C., Using simulation to improve the blood supply chain. J. Oper. Res. Soc. 58(2), (2007), pp. 219-227

10. Hemmelmayr, Vera. Doerner, Karl F. Hartl, Richard F. Savelsbergh, Martin W. P.(2008). Delivery Strategies for Blood Products Supplies, OR Spectr. 31, 707-725. 
11. Sivakumar, P. Ganesh, K. Parthiban, P. (2008). Multi-phase composite analytical model for integrated allocation-routing problem - application of blood bank logistics

12. Haijema, R., van Dijk, N., van der Wal, J., Smit Sibinga, C., 2009. Blood platelet production with breaks: optimization by SDP and simulation. International Journal of Production Economics 121, 464-473.

13. Xin, L(2010). Simulation and Optimization Research ofBlood Center Inventory Management under Dynamic Demand. International $\mathrm{C}$ onference on Future Information Tec hnology and Management Engineering

14. Nagurney, Anna, Amir H Masoumi, and Yu Min. 2012. "Supply Chain Network Operations Management of a Blood Banking System with Cost and Risk Minimization." Computational Management Science 9 (2): 205-231.

15. Alfonso, E., 695 Xie, X., Augusto, V., Garraud, O., 2013. Modelling and simulation of blood collection systems: improvement of human resources allocation for better cost-effectiveness and reduction of candidate donor abandonment. Vox Sanguinis $104,225-233$.

16. Baesler, F., Nemeth, M., Martínez, C., Bastías, A., 2014. Analysis of inventory strategies for blood components in a regional blood center using process simulation. Transfusion 54, 323-30.

17. Ganesh, K., Narendran, T.T., Anbuudayasankar, S.P., 2014. Evolving cost-effective routing of vehicles for blood bank logistics. International Journal of Logistics Systems and Management 17, 381-415.

18. Guðbjörnsdóttir, Elísabet Edda(2015). Blood Bank Inventory Management Analysis, Thesis of Master of Science in Engineering Management

19. Puranam, K. Novak, D. C. Lucas, M. T. Fung, M. Managing blood inventory with multiple independent sources of supply. European Journal of Operational Research, 259(2), (2017), pp. 500-511.

20. Khaldi, R. Abedllatif, E.A. Raddouane, Ch. Rduan, F. Artificial neural network based approach for blood demand forecasting: Fez transfusion blood center case 
study. Proceedings of the 2nd international conference in Big Data, Cloud and Applications Article, No. 59, 2017

21. Ahmadimanesh M, Tavakoli A, Pooya A, Dehghanian F.Designing an optimal inventory management model for the blood supply chain: synthesis of reusable simulation and neural network. Medicine 2020;99:29 (e21208).

22. Kazemi(a), Seyed Mahmood. Rabbani, Masoud. Tavakkoli-Moghaddam, Reza. Abolhassani SHAHREZA, Farid (2017). AN EXACT SOLUTION FOR JOINT OPTIMIZATION OF INVENTORY AND ROUTING DECISIONS IN BLOOD SUPPLY CHAINS: A CASE STUDY, Economic Computation and Economic Cybernetics Studies and Research, Issue 4/2017; Vol. 51

23. Kazemi(b), Seyed Mahmood. Rabbani, Masoud. Tavakkoli-Moghaddam, Reza(2017). Blood inventory-routing problem under uncertainty, Journal of Intelligent \& Fuzzy Systems 32 (2017) 467-481

24. Babagolzade, M. pirayesh, M.A. Salari, M.(2014). Provide an inventory-routing model for perishable goods. Forty-fifth Iranian Mathematical Conference, 2014. 
Figures

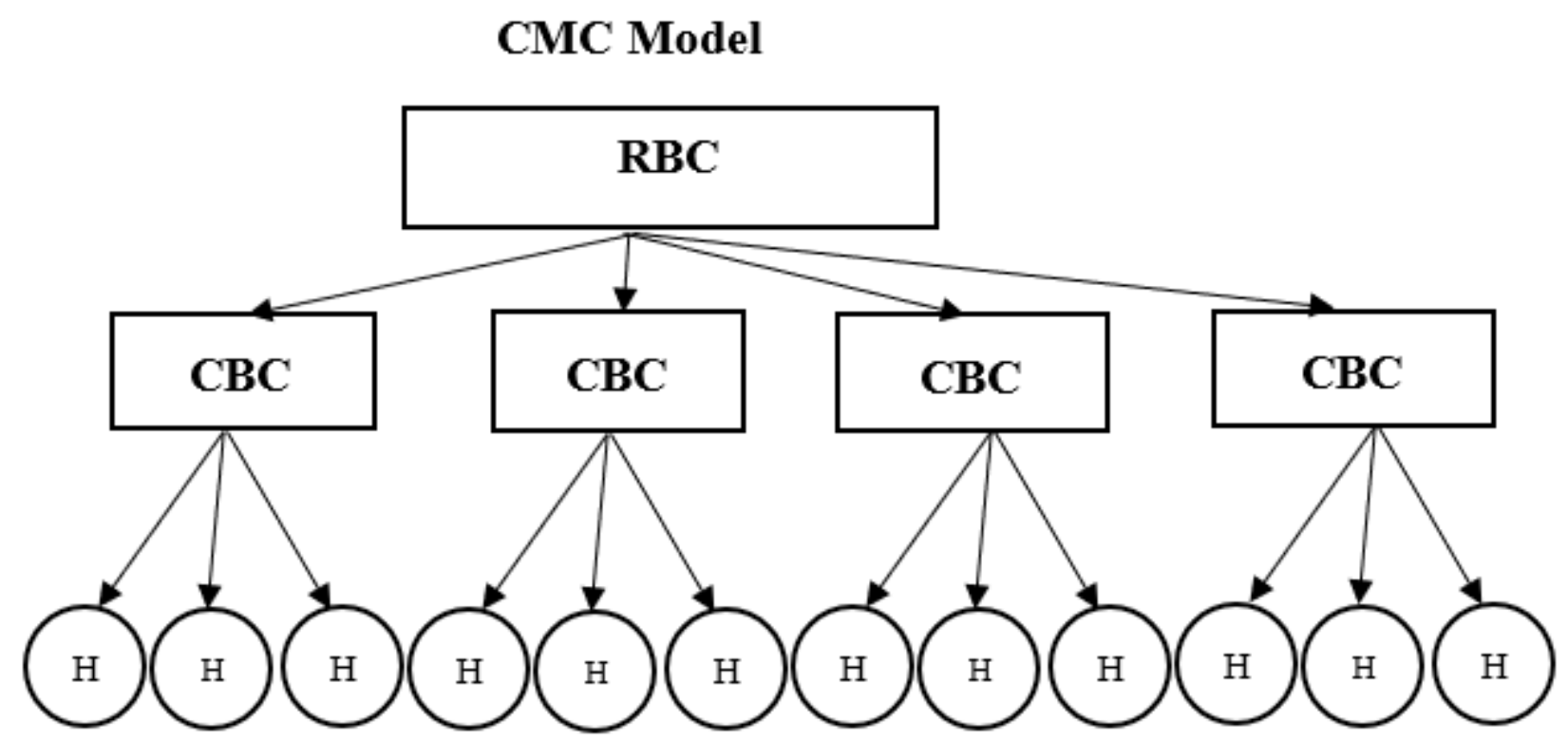

Figure 1

Center structure of the studied region 


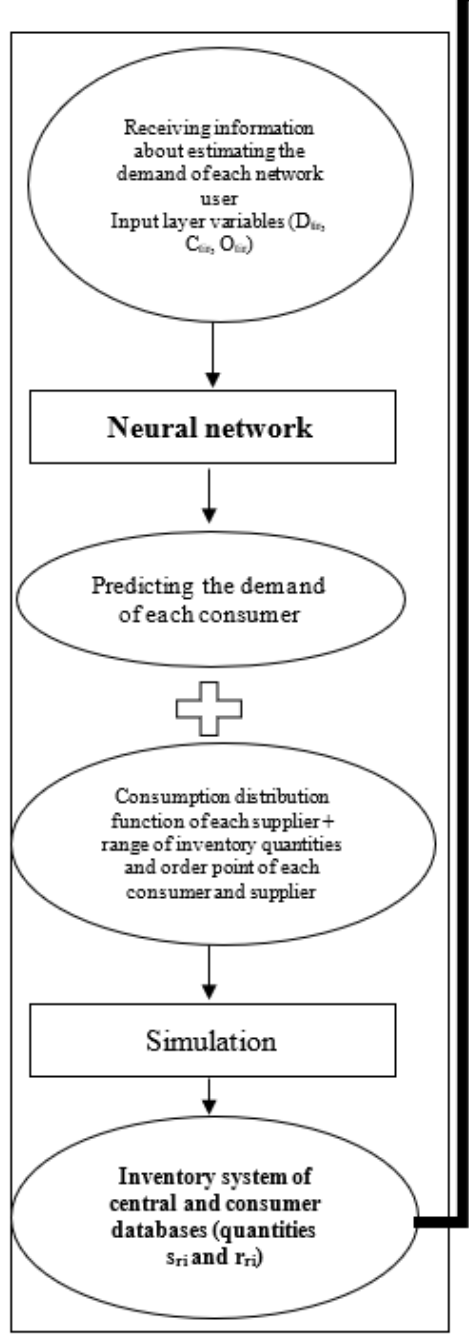

Preparing RBC level instructions for CBC level

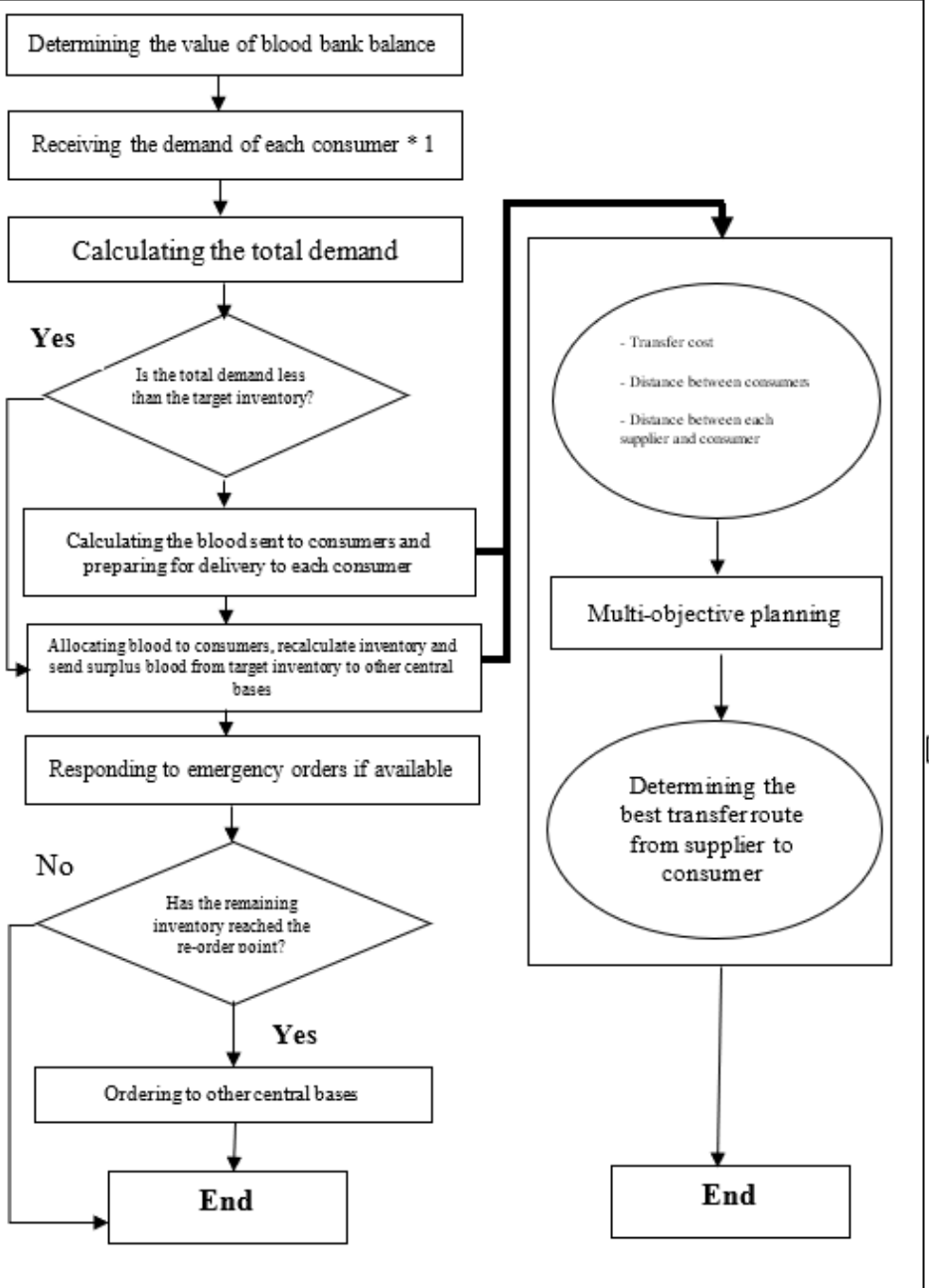

CBC level planning on day $t$

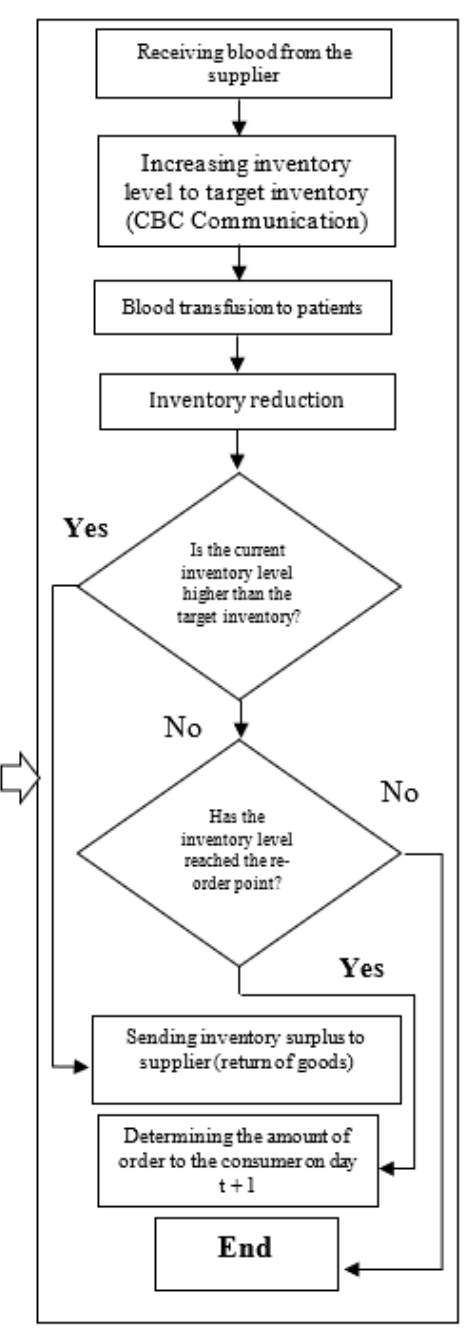

Hospital level planning on day $t$

Figure 2

Research algorithm 


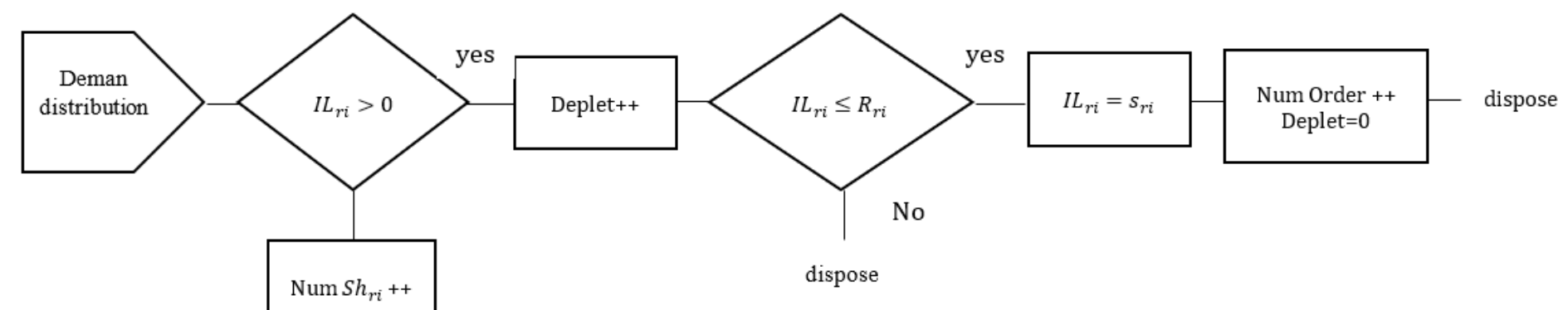

No

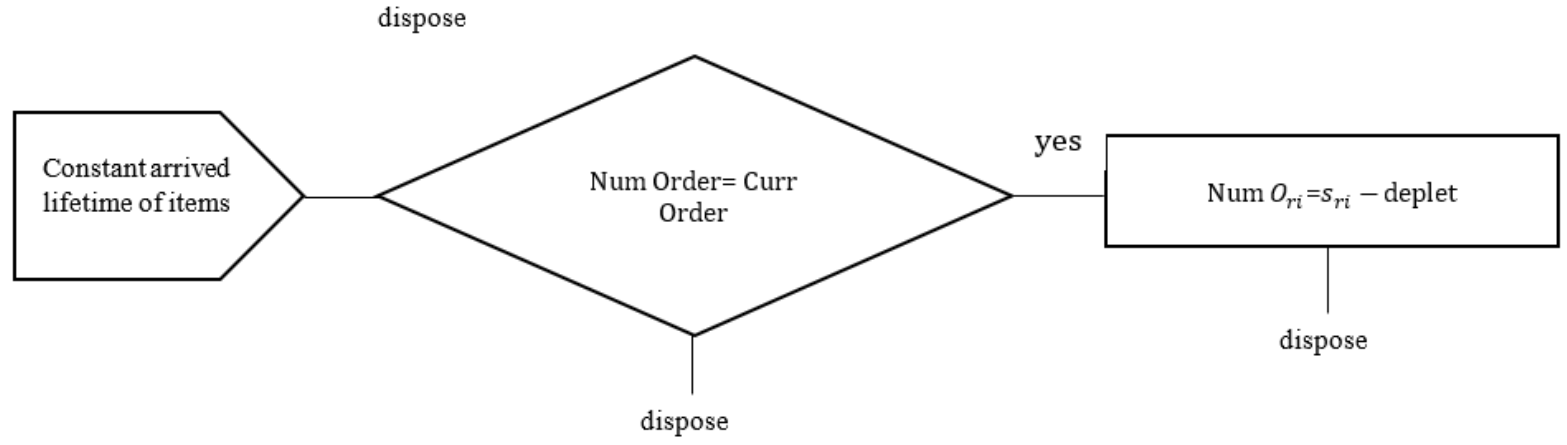

Figure 3

Simulation algorithm

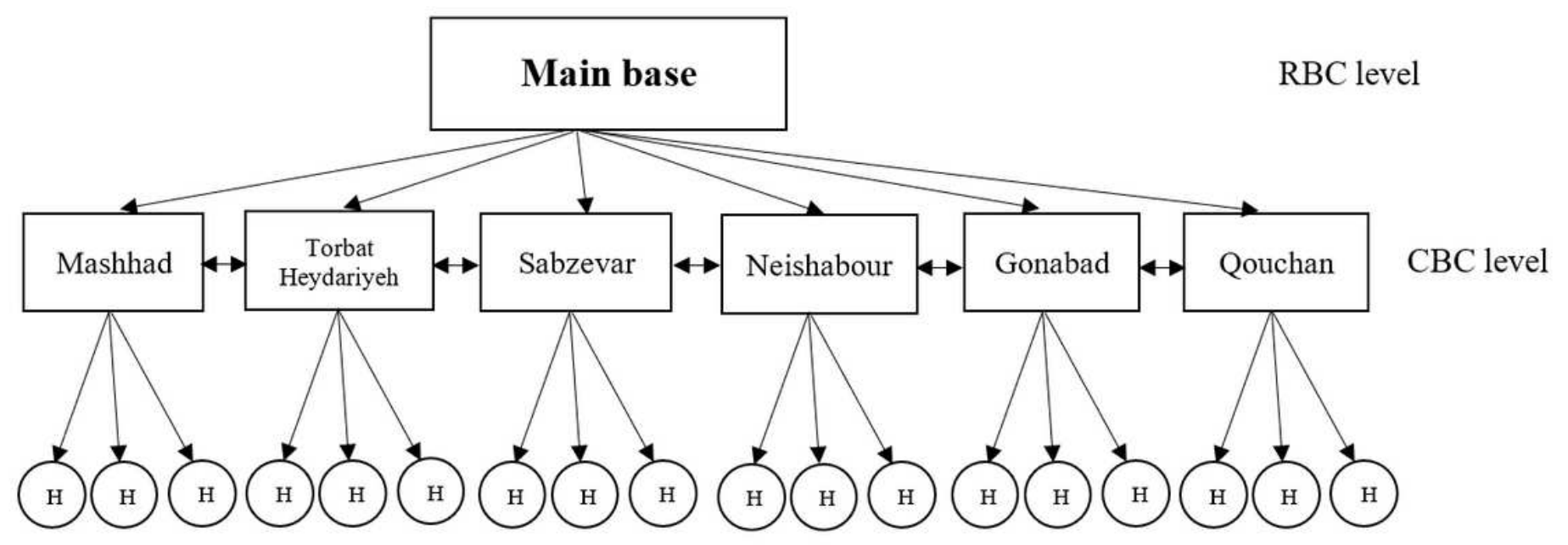

Figure 4

Regional blood structure of Khorasan Razavi blood transfusion network

Figure 5 
The values of actual demand and blood transfusion to Mashhad hospitals of Mashhad Central Base by blood group

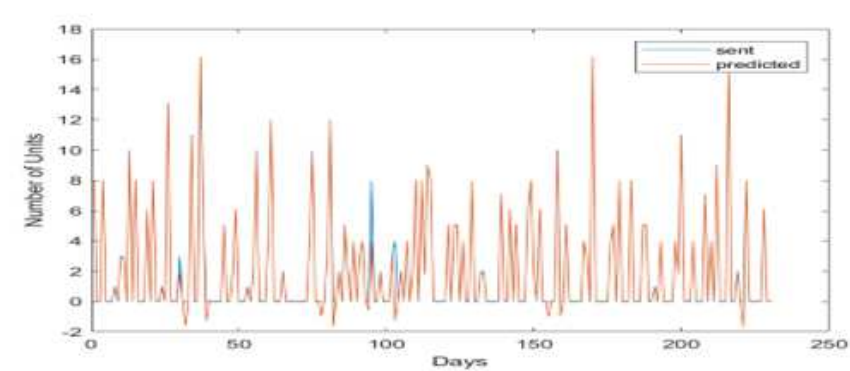

A

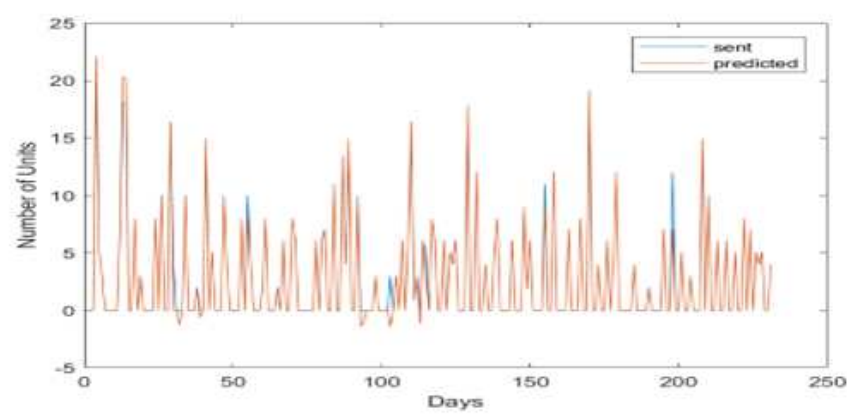

$\mathrm{O}$

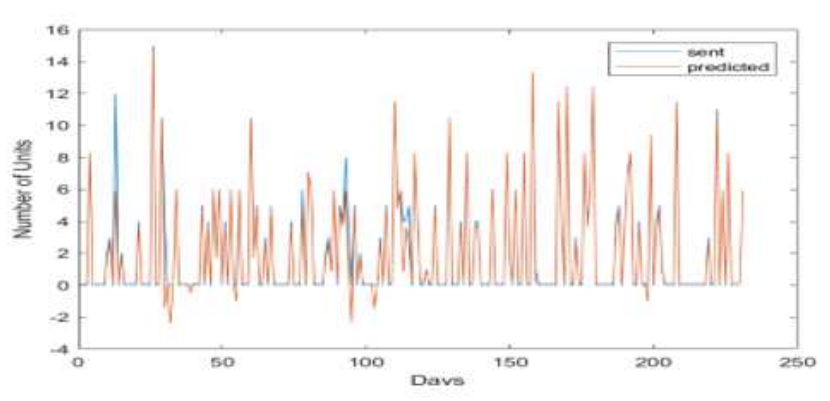

B

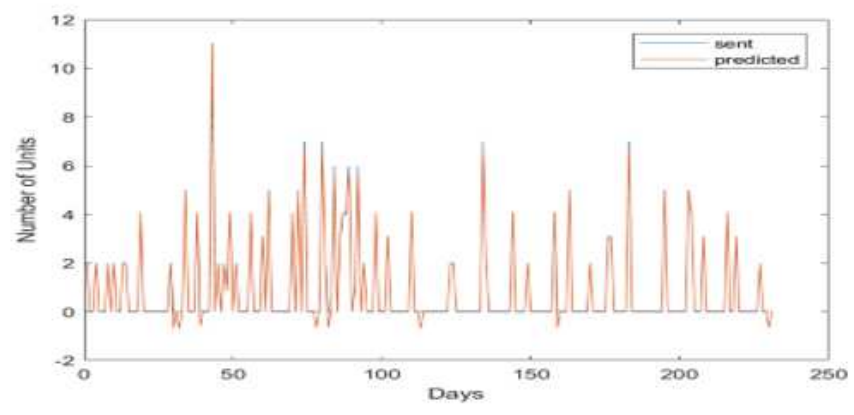

$\mathrm{AB}$

Figure 6

Actual blood demand and sent rates to Aria Hospital by blood type

\section{Supplementary Files}

This is a list of supplementary files associated with this preprint. Click to download.

- tables.docx 